\title{
Feature Level Fusion of Face and Palmprint Biometrics
}

\author{
Dakshina Ranjan Kisku ${ }^{1}$, Phalguni Gupta ${ }^{2}$, and Jamuna Kanta Sing ${ }^{3}$ \\ ${ }^{1}$ Department of Computer Science and Engineering, \\ Dr. B. C. Roy Engineering College, Durgapur - 713206, India \\ ${ }^{2}$ Department of Computer Science and Engineering, \\ Indian Institute of Technology Kanpur, Kanpur - 208016, India \\ ${ }^{3}$ Department of Computer Science and Engineering, \\ Jadavpur University, Kolkata - 700032, India \\ \{drkisku,jksing\}@ieee.org, pg@cse.iitk.ac.in
}

\begin{abstract}
This paper presents a feature level fusion of face and palmprint biometrics. It uses the improved K-medoids clustering algorithm and isomorphic graph. The performance of the system has been verified by two distance metrics namely, K-NN and normalized correlation metrics. It uses two multibiometrics databases of face and palmprint images for testing. The experimental results reveal that the feature level fusion with the improved K-medoids partitioning algorithm exhibits robust performance and increases its performance with utmost level of accuracy.
\end{abstract}

Keywords: Biometrics, Feature Level Fusion, Face, Palmprint, Isomorphic Graph, K-Medoids Partitioning Algorithm.

\section{Introduction}

Feature level fusion fuses feature sets of different biometric traits under different fusion rules. Since feature level fusion integrates richer and most relevant information of biometric evidences and it is expected to provide more accurate authentication results. It is found to be effective compared to fusion based on match scores, decision, ranks, etc. But fusion of incompatible biometric evidences at feature level is a very hard task. Moreover, the feature spaces for different biometric evidences may be unknown and this may lead to the problem of curse of dimensionality [1]. Also, poor feature representation may degrade the performance of recognition.

Unibiometric identifiers are often affected by problems like lack of invariant representation, non-universality, noisy sensor data and lack of individuality of the biometric trait and susceptibility to circumvention. These problems can be minimized by using multibiometric systems that consolidate evidences obtained from multiple biometric sources. Multibiometrics fusion at match score level, decision level and rank level have extensively been studied while there exists a few feature level fusion approaches. There is enough scope to design an efficient feature level fusion approach. The feature level fusion of face and palmprint biometrics proposed in [3] uses single sample of each trait. Discriminant features using graph-based approach and principal component analysis techniques are used to extract features from face and palmprint. 
Further, a distance separability weighing strategy is used to fuse two sets at feature extraction level. Another approach consisting of face and hand biometrics has been proposed in [4]. In [5], a feature level fusion has been studied where phase congruency features are extracted from face and Gabor transformation is used to extract features from palmprint. These two feature spaces are then fused using user specific weighting scheme. Another approach of face and palmprint biometrics is given in [6]. It makes use of correlation filter bank with class-dependence feature analysis method for feature fusion.

This paper proposes a feature level fusion of face [7] and palmprint [8] biometrics using isomorphic graph [9] and K-medoids [10]. SIFT feature points [11] are extracted from face and palmprint images. The partitioning around medoids (PAM) algorithm [12] is used to partition the face and palmprint images of a set of $n$ invariant feature points into $k$ number of clusters. For each cluster, an isomorphic graph is drawn on SIFT points belonging to the clusters. Graphs are drawn on each cluster by searching the most probable isomorphic graphs using iterative relaxation algorithm [13] from all possible isomorphic graphs while the graphs are compared between face and palmprint templates. Each pair of clustered graphs are then fused by concatenating the invariant SIFT points and all pairs of isomorphic graphs of clustered regions are further fused to make a single concatenated feature vector. The similar feature vector is also constructed from query pair of face and palmprint. Finally, matching between these two vectors is done by computing the distance using K-Nearest Neighbor [14] and normalized correlation [15] distance. Two multimodal databases are used for testing the proposed technique.

The paper is organized as follows. Next section discusses SIFT features extraction from face and palmprint images. Section 3 presents K-Medoids partitioning of SIFT features into a number of clusters. The method of obtaining isomorphic graphs on the sets of the SIFT points which belong to the clusters is also discussed. Next section presents feature level fusion of clustered SIFT points by pairing two graphs of a pair of clustered regions drawn on face and palmprint images. Experimental results and a comparative study are presented in Section 5 while conclusion is made in the last section.

\section{SIFT Keypoints Extraction}

David Lowe [11] has proposed a technique to extract features from images which are called Scale Invariant Feature Transform (SIFT). These features are invariant to scale, rotation, partial illumination and 3D projective transform. SIFT provide a set of features of an object that are not affected by occlusion, clutter and unwanted noise in the image. In addition, the SIFT features are highly distinctive in nature which have accomplished correct matching on several pair of feature points with high probability between a large database and a test sample. Initially, the face and palmprint images are normalized by adaptive histogram equalization [1]. Localization of face is done by the face detection algorithm proposed in [16] while the algorithm in [17] is used to localize palmprint. SIFT features [11] are extracted from the face and palmprint images. Each feature point is composed of four types of information - spatial location $(x, y)$, scale $(S)$, orientation $(\theta)$ and Keypoint descriptor $(K)$. It uses only keypoint 
descriptor which consists of a vector of 128 elements showing change in neighborhood intensity of each keypoint. Local image gradients are measured at the selected scale in the region around each keypoint. These gradients are then transformed into a vector that contains 128 elements. These vectors represent local shape distortions and illumination changes.

\section{Feature Partitioning and Isomorphic Graph Representation}

Lack of well feature representation often degrades the performance. A well representation of feature space and template in terms of invariant feature points may help to increase the overall performance of the system. Clustering of all SIFT feature points into a number of clusters with limited number of invariant points can be an efficient approach of feature space representation. Clustering approach [18] gathers together the keypoints found to be more relevant members of a particular cluster.

\subsection{SIFT Keypoints Partitioning}

$\mathrm{K}$-medoids clusters is an adaptive version of $\mathrm{K}$-means clustering approach and is used to partition the dataset into a number of groups which minimizes the squared error between the points that belong to a cluster and a point designated as the center of the cluster. The generalization of K-medoids algorithm is the Partitioning around Medoids (PAM) algorithm [12] which is applied to the SIFT keypoints of face and palmprint images to obtain the partitioned of features which can provide more discriminative and meaningful clusters of invariant features.

After applying the PAM clustering technique [12] to the sets of SIFT keypoints for face and palmprint images, each cluster can be verified by Silhouette technique. For each keypoint, let $i, x(i)$ be the average distance of $i$ with all the keypoints in cluster $c_{m}$. Consider $x(i+1)$ is an average distance next to $x(i)$. These two successive distances $x(i)$ and $x(i+1)$ are considered to verify the matching of these keypoints $i$ and $(i+1)$ to the cluster where these points are assigned. Then the average distances of $i$ and $(i+1)$ with the keypoints of another single cluster are found. Repeat this process for every cluster in which $i$ and $(i+1)$ are not a member. If the cluster with lowest average distances to $i$ and $(i+1)$ are $y(i)$ and $y(i+1),(y(i+1)$ is the next lowest average distance to $y(i))$, the cluster is known to be the neighboring cluster of the former cluster in which $i$ and $(i+1)$ are assigned. It can be defined by

$$
S(i)=\frac{(y(i)+y(i+1)) / 2-(x(i)+x(i+1)) / 2}{\max [((x(i)+x(i+1)),(y(i)+y(i+1))]}
$$

From Equation (1) it can be written that $-1 \leq S(i) \leq 1$. When $x(i)+x(i+1)<<$ $y(i)+y(i+1), S(i)$ would be close to 1 . Distances $x(i)$ and $x(i+1)$ are the measures of dissimilarity of $i$ and $(i+1)$ to its own cluster. If $y(i)+y(i+1)$ is small, then it is well matched; otherwise when the value of $y(i)+y(i+1)$ is large then match is bad. Keypoint is well clustered when $S(i)$ is closer to 1 and when $S(i)$ is negative then it belongs to another cluster. $S(i)$ is zero for the keypoint on the border of any two clusters. The existing algorithm has been extended by taking average distances between $x(i+1)$ 
and $y(i+1)$ for a pair of clusters and a better approximation could be found while PAM algorithm is used to partition the keypoints. The precision of each cluster is increased by this approximation where more relevant keypoints instead of restricted number of keypoints for fusion are taken.

\subsection{Establishing Correspondence}

To establish correspondence between any two clusters of face and palmprint images, it is observed that more than one keypoint on face image may correspond to single keypoint on the palmprint image. To eliminate false matches and to consider only minimum pair distance from a set of pair distances for making correspondences, it needs to verify the number of feature points available in the cluster of face and that in the palmprint cluster. When the number of feature points in the cluster for face is less than that of the palmprint cluster, many points of interest from the palmprint cluster need to be discarded. If the number of points of interest on the face cluster is more than that of the palmprint cluster, then a single interest point on the palmprint cluster may act as a match point for many points of interest of face cluster. Also, many points of interest on the face cluster may have correspondences to a single point of interest on the palmprint cluster. From all such making correspondences, minimum distance pair is paired. Isomorphic graph for each cluster has been formed by removing few more keypoints from the paired clusters. Iterative relaxation algorithm [13] is used for searching the best possible pair of isomorphic graphs from all possible graphs.

\subsection{Isomorphic Graph Representations}

To interpret each pair of clusters for face and palmprint, isomorphic graph representation has been used. Each cluster contains a set of SIFT keypoints [11] and each keypoint is considered as a vertex of the proposed isomorphic graph. A one-to-one mapping function is used to map the keypoints of the isomorphic graph constructed on a face cluster to a palmprint cluster while these two clusters have been made correspondence to each other. When two isomorphic graphs are constructed on a pair of face and palmprint clusters with equal number of keypoints, two feature vectors of keypoints are found for fusion. Let $F_{G}$ and $P_{G}$ be two graphs and also let $f$ be a mapping function from the vertex set of $F_{G}$ to vertex set of $P_{G}$. So when $f$ is one-to-one and $f\left(v_{k}\right)$ is adjacent to $f\left({ }_{w k}\right)$ in $P_{G}$ if and only if $v_{k i}$ s adjacent to $w_{k} n F_{G}$, the function $f$ is known as an isomorphism and two graphs $F_{G}$ and $P_{G}$ are isomorphic.

\section{Fusion of Keypoints and Matching}

\subsection{Fusion of Keypoints}

To fuse the SIFT keypoint descriptors obtained from each isomorphic pair of graphs for face and for palmprint images, two different fusion rules are applied serially, viz. sum [2] and concatenation [1] rules. Let $F_{G}\left(v_{k}\right)=\left(v_{k l}, v_{k 2}, . ., v_{k n}\right)$ and $P_{G}\left(w_{k}\right)=\left(w_{k l}\right.$, $\left.w_{k 2}, \ldots, w_{k n}\right)$ be the two sets of keypoints obtained from two isomorphic graphs for a pair of face and palmprint clusters. Suppose there are $m$ numbers of clusters in each of face and palmprint images. These two sets of clusters are fused using sum rule and the 
concatenation rule is further applied to form an integrated feature vector. Suppose, $F_{G 1}, F_{G 2}, \ldots, F_{G m}$ sets of keypoints are obtained from a face image after clustering and isomorphism and $P_{G 1}, P_{G 2}, \ldots, P_{G m}$ are the sets of keypoints obtained from a palmprint image. The sum rule for the fusion of keypoints is as follows

$$
\begin{aligned}
& S_{F P 1}=F_{G 1}+P_{G 1}=\left\{\left(v_{k 1}^{1}+w_{k 1}^{1}\right),\left(v_{k 2}^{1}+w_{k 2}^{1}\right),\left(v_{k 3}^{1}+w_{k 3}^{1}\right), \ldots,\left(v_{k n}^{1}+w_{k n}^{1}\right)\right\} \\
& S_{F P 2}=F_{G 2}+P_{G 2}=\left\{\left(v_{k 1}^{2}+w_{k 1}^{2}\right),\left(v_{k 2}^{2}+w_{k 2}^{2}\right),\left(v_{k 3}^{2}+w_{k 3}^{2}\right), \ldots,\left(v_{k n}^{2}+w_{k n}^{2}\right)\right\} \\
& \begin{array}{ccccccccccc}
----- & ---- & ---- & ---- & ----- & ---- & ---- & ---- & ---- & ----- & ---- \\
---- & ---- & ---- & ---- & ---- & ---- & ---- & ---- & ---- & ---- & ----
\end{array} \\
& S_{F P m}=F_{G m}+P_{G m}=\left\{\left(v_{k 1}^{m}+w_{k 1}^{m}\right),\left(v_{k 2}^{m}+w_{k 2}^{m}\right),\left(v_{k 3}^{m}+w_{k 3}^{m}\right), \ldots,\left(v_{k n}^{m}+w_{k n}^{m}\right)\right\}
\end{aligned}
$$

where $v_{k j}(j=1,2, \ldots, n)$ and $w_{k j}(j=1,2, \ldots, n)$ of $S_{F P j}(i=1,2, . ., m)$ refer to a keypoint of a face graph and a keypoint of a palm graph respectively. In the next step, concatenation rule is applied to the sets of keypoints to form a single feature vector.

\subsection{Matching Criterion and Verification}

The K-Nearest Neighbor (K-NN) distance [14] and correlation distance [15] approaches are used to compute distances from the concatenated feature sets. In K-NN approach, Euclidean distance metric is used to get $K$ best matches. Let $d_{i}$ be the Euclidian distance of the concatenated feature set of subject $S_{i}, i=1,2, \ldots . K$, which belong to the $K$ best matches against a query subject. Then $S_{t}$ is verified against the query if $d_{t} \leq T h$ where $d_{t}$ is the minimum of $d_{l}, d_{2}, \ldots, d_{K}$ and $T h$ is the threshold.

On the other hand, the correlation distance metric is used for computing distance between a pair of reference set and probe set. Similarity between two concatenated feature vectors $f_{1}$ and $f_{2}$ can be computed as follows

$$
d=\frac{\sum f_{1} f_{2}}{\sqrt{\sum f_{1} \sum f_{2}}}
$$

Equation (3) denotes the normalized correlation between feature vectors $f_{1}$ and $f_{2}$. Let $d_{i}$ be the similarity of the concatenated feature set of subject $S_{i}, i=1,2, \ldots K$, with respect to that of a query subject. Then the subject $S_{t}$ is verified against the query subject if $d_{t} \geq T h$ where $d_{t}$ is the maximum of $d_{1}, d_{2}, \ldots, d_{K}$ and $T h$ is the threshold.

\section{Experimental Evaluation and Databases}

\subsection{Databases}

The proposed approach has been tested on IIT Kanpur and chimeric multimodal databases. Chimeric database contains face images of ORL face database [20] and palmprint images of Hong Kong Polytechnic University (PolyU) database [21]. IIT Kanpur multibiometrics database consists of 800 face and 800 palmprint images and each subject contributes 2 face and 2 palmprint images. ORL face database contains 400 face images of 40 subjects while PolyU database contains 7,752 palmprint images of 193 subjects (386 palm impressions). From 400 face images of ORL database [20], 
only 160 face images are taken and 4 face images are taken for each subject. From PolyU database [21], only 160 palm images of 40 subjects having 2 right and 2 left palm images per subject are taken.

In IIT Kanpur face database, images are in controlled environment with maximum tilt of head by $20^{\circ}$ from the origin. For evaluation, frontal view faces are used with uniform lighting and minor change in facial expression. These images are acquired in two different sessions. Among the two face images, one image is used as a reference face and the other one is used as a probe face. After preprocessing of face images, it uses the face detection algorithm [16] to get face portion only. On the other hand, face images in ORL database [20] are taken at different sessions with varying the lighting conditions, facial expressions (open/closed eyes, smiling/not smiling) and different facial details (glasses / no glasses). The face images are taken against a dark homogeneous background with the subjects in an upright, frontal position. For the experiment, only frontal view faces are taken with neutral facial expressions and uniform changes in lighting. Among the 4 face images, 2 images are used for reference and remaining two are used for probe face images. Since it contains cropped images, one does not require getting the face portion.

Palmprint images in IIT Kanpur database are also taken in controlled environment with a flat bed scanner having spatial resolution of $200 \mathrm{dpi}$. Impressions are taken on the scanner with rotation of at most $\pm 35^{\circ}$ to each user. There are 800 palmprint images of 400 subjects and each subject is contributed 2 images. An image enhancement technique is used to achieve uniform spatial resolution. Finally, palm portion is detected with the help of the technique proposed in [17]. In PolyU palmprint database [21], images are captured at two different sessions and these images are taken under different lighting conditions and by changing the focus of CCD camera. Change in focus is regarded as different palm capturing devices. The images which are of two different sizes, viz. $384 \times 284$ and $768 \times 568$, are resized to $160 \times 160$ and palm portion is detected by the algorithm presented in [17].

\subsection{Experimental Results}

The performance of the proposed approach is determined using one-to-one matching strategy. Experimental results are obtained with the help of two distance approaches namely, K-Nearest Neighbor (K-NN) distance [14] and normalized correlation [15]. We have also determined the performance of face and palmprint independently. Fused feature set which is obtained from reference face and palmprint images is matched with the feature set obtained from probe pair of face and palmprint images by computing the distance between these two sets. Experiments are for the six distinct cases: (i) face modality using K-NN, (ii) face modality using normalized correlation, (iii) palmprint modality using K-NN, (iv) palmprint modality using normalized correlation, (v) feature fusion using K-NN and (vi) feature fusion using normalized correlation.

False Accept Rate (FAR), False Reject Rate (FRR) and recognition rate are determined from the IIT Kanpur database of 800 face and palmprint images of 400 subjects. Feature level fusion method using normalized correlation outperforms other proposed methods including individual matching of face and palmprint modalities. The correlation metric based feature level fusion has $98.75 \%$ recognition rate with $0 \%$ 
FAR while K-NN based method has the recognition rate of $97.5 \%$ with $2 \%$ FAR. It can be noted that FAR of all the proposed methods are found to be less than its corresponding FRR. On the other hand, palmprint modality performs better than face modality while K-NN and correlation metrics are used. The distance metrics play an important role irrespective of use of invariant features and isomorphic graphs representations. However, the robust representation of face and palmprint images using isomorphic graphs with use of invariant SIFT keypoints and PAM characterized KMedoids algorithm makes the proposed fusion method more efficient. In single modality, the same approach has been used. Therefore, the error rates obtained from the single modalities and fusion method are determined under a uniform framework. However, the methodology used for feature level fusion found to be not only superior to other methods and also shows significant improvements in terms of recognition rate and FAR. Table 1 shows different error rates determined on IIT Kanpur database for the methods

Table 1. Different Error Rates on IIT Kanpur Database

\begin{tabular}{llc}
\hline \multicolumn{1}{c}{ METHOD } & FAR (\%) & $\begin{array}{l}\text { RECOGNITION } \\
\text { RATE (\%) }\end{array}$ \\
\hline Face Recognition (K-NN) & 7.0 & 92.50 \\
Face Recognition (Correlation) & 6.0 & 93.75 \\
Palmprint Verification (K-NN) & 4.5 & 94.75 \\
Palmprint Verification (Correlation) & 2.5 & 96.00 \\
Feature Level Fusion (K-NN) & 2.0 & 97.50 \\
Feature Level Fusion (Correlation) & 0.0 & 98.75 \\
\hline
\end{tabular}

In the second phase, when the proposed fusion is applied with both the correlation based and K-NN based distance metrics for the chimeric multibiometric database, the FAR is found to be much lesser than that of FRR. The correlation based distance metric has $99.5 \%$ recognition rate with $0 \%$ FAR while the K-NN distance metric has $99.25 \%$ recognition rate with $1.5 \%$ FAR. It is found that the palmprint modality performs better than face modality under both the distance metrics. The combination of SIFT features and isomorphic graph representation is found to be robust for the proposed feature level fusion approach while the IIT Kanpur and chimeric multibiometric databases are evaluated. However, the recognition rates determined from chimeric database is found to be more than that of IIT Kanpur database. This is because of the small size compared to IIT Kanpur database. Table 2 shows the error rates and recognition rates for the proposed techniques on chimeric database.

Table 2. Error and Recognition Rates Determined on Chimeric Database

\begin{tabular}{lll}
\hline \multicolumn{1}{c}{ METHOD } & FAR (\%) & $\begin{array}{l}\text { RECOGNITION } \\
\text { RATE }(\%)\end{array}$ \\
\hline Face Recognition (K-NN) & 5.5 & 93.75 \\
Face Recognition (Correlation) & 5 & 94.5 \\
Palmprint Verification (K-NN) & 4 & 95 \\
Palmprint Verification (Correlation) & 2.25 & 96.75 \\
Feature Level Fusion (K-NN) & 1.5 & 99.25 \\
Feature Level Fusion (Correlation) & 0.0 & 99.5 \\
\hline
\end{tabular}


Sub-graph isomorphism is robust and optimal routing representation where most of the feature points construct good representative graph for the other biometric sample on which the feature points of the first graph is mapped. This characteristic of subgraph isomorphism makes the feature level fusion more robust.

Table 3. Best Recognition Accuracies for Proposed Fusion and for Fusion Approach in [19]

\begin{tabular}{|c|c|c|c|}
\hline METHOD & DATABASE & $\begin{array}{l}\text { NUMBER OF } \\
\text { FEATURE POINTS }\end{array}$ & $\mathrm{RR}(\%)$ \\
\hline $\begin{array}{l}\text { Feature level fusion } \\
\text { [Experiment I] [19] }\end{array}$ & $\begin{array}{l}\text { Local database ( } 480 \text { faces, } \\
120 \text { hand geometry, } 30 \\
\text { individuals) }\end{array}$ & $\begin{array}{l}21 \text { points ( } 8 \text { points } \\
\text { from eyes, } 4 \text { points } \\
\text { from nose and } 9 \\
\text { points from hand) }\end{array}$ & 99.23 \\
\hline $\begin{array}{l}\text { Feature level fusion } \\
\text { [Experiment I] [19] }\end{array}$ & $\begin{array}{l}\text { Local database ( } 480 \text { faces, } \\
120 \text { hand geometry, } 30 \\
\text { individuals) }\end{array}$ & $\begin{array}{l}25 \text { points ( } 16 \text { points } \\
\text { from eyes, and } 9 \\
\text { points from hand) }\end{array}$ & 99.22 \\
\hline $\begin{array}{l}\text { Feature level fusion } \\
\text { [Experiment II] [19] }\end{array}$ & $\begin{array}{l}\text { Local database ( } 480 \text { faces, } \\
120 \text { hand geometry, } 30 \\
\text { individuals) }\end{array}$ & $\begin{array}{l}21 \text { points ( } 8 \text { points } \\
\text { from eyes, } 4 \text { points } \\
\text { from nose and } 9 \\
\text { points from hand) }\end{array}$ & 99.43 \\
\hline $\begin{array}{l}\text { Feature level fusion } \\
\text { [Experiment II] [19] }\end{array}$ & $\begin{array}{l}\text { Local database ( } 480 \text { faces, } \\
120 \text { hand geometry, } 30 \\
\text { individuals) }\end{array}$ & $\begin{array}{l}21 \text { points ( } 8 \text { points } \\
\text { from eyes, } 4 \text { points } \\
\text { from nose and } 9 \\
\text { points from hand) }\end{array}$ & 99.31 \\
\hline $\begin{array}{l}\text { Feature Level } \\
\text { Fusion (K-NN) }\end{array}$ & $\begin{array}{l}\text { IIT Kanpur ( } 800 \text { faces, } \\
800 \text { palms, } 400 \\
\text { individuals) }\end{array}$ & $\begin{array}{l}\text { Feature points are } \\
\text { not fixed }\end{array}$ & 97.5 \\
\hline $\begin{array}{l}\text { Feature Level } \\
\text { Fusion (Correlation) }\end{array}$ & $\begin{array}{l}\text { IIT Kanpur ( } 800 \text { faces, } \\
800 \text { palms, } 400 \\
\text { individuals) }\end{array}$ & $\begin{array}{l}\text { Feature points are } \\
\text { not fixed }\end{array}$ & 98.75 \\
\hline $\begin{array}{l}\text { Feature Level } \\
\text { Fusion (K-NN) }\end{array}$ & $\begin{array}{l}\text { Chimeric ( } 160 \text { faces, } 160 \\
\text { palms, } 40 \text { individuals) }\end{array}$ & $\begin{array}{l}\text { Feature points are } \\
\text { not fixed }\end{array}$ & 99.25 \\
\hline $\begin{array}{l}\text { Feature Level } \\
\text { Fusion (Correlation) }\end{array}$ & $\begin{array}{l}\text { Chimeric ( } 160 \text { faces, } 160 \\
\text { palms, } 40 \text { individuals) }\end{array}$ & $\begin{array}{l}\text { Feature points are } \\
\text { not fixed }\end{array}$ & 99.5 \\
\hline
\end{tabular}

\subsection{Comparison with Other Technique}

The proposed fusion of face and palmprint is compared with a multibiometrics system [19] where the features of face and hand evidences are fused. In the proposed fusion, SIFT features are extracted from face and palmprint and on these feature points, isomorphic graphs are drawn. These isomorphic representations are fused in terms of matched points found on isomorphic subgraphs. On the other hand, in [19] local facial features, such as eyes, mouth and nose features are localized using point distribution model and active shape models. Similarly, same methodology is applied to find some distinctive points on hand geometry. Gabor filter is applied to face image and feature vector is constructed by extracting the key points using active shape models. Similarly the hand feature vector is constructed. To verify the identity of users, Support Vector Machine is used. The technique presented in [19] is tested on a multibiometrics database which contains 480 face images and 120 hand images of 30 peoples. 16 faces and 4 hand images are taken from each person. Two experiments are conducted on the 
entire database. In the first experiment, features of 12 faces and 2 hands are fused for training and for testing, feature of 4 faces and remaining 2 hands are fused. The system is trained on 12 feature vectors which contain information about face and hand geometry of each individual. One SVM is trained on each individual. In this experiment numbers of hand features are fixed to every combination of features, where the number of features for eyes, nose and mouth are changing in every combination. The best recognition accuracy obtained from the first experiment is $99.23 \%$. In the second experiment features of 12 faces and 3 hands are fused. This combination achieves best average accuracy while the system is trained with SVM. The best average accuracy obtained by the feature vector which contains Gabor features of 8 eye points, 4 nose points and 9 hand geometry. Table 3 shows the best average accuracy of different combinations of feature points. The best average recognition accuracy obtained from the second experiment is $99.43 \%$.

The proposed approach shows the best recognition accuracies (RR) on IIT Kanpur and chimeric databases. Test on IIT Kanpur reveals $98.75 \%$ and $97.5 \%$ accuracies under normalized correlation and K-NN distance metrics respectively. In case of the chimeric database, they are $99.5 \%$ and $99.25 \%$. The accuracies of the proposed approach are better than that of the approach in [19]. Since, the number of invariant features on both the face and palmprint images is not fixed, the performance shows outmost level of robust system. However, the fusion approach in [19] takes fixed number of features obtained from eyes, mouth and nose. And some distinctive features are determined from hand geometry. Number of SIFT feature points in the proposed fusion is changed dynamically and the combination of subgraph isomorphism and SIFT descriptor exhibits robustness of the fusion system. The system in [19] shows certain variations in selection of local feature points and it also shows good accuracies. However, due to fixed number of feature points and number of less feature points exhibit robustness to some extent. In the proposed fusion the whole face is used for feature extraction while the fusion approach in [19] uses the local features only.

\section{Conclusion}

This paper has proposed a feature level fusion approach of face and palmprint biometrics using invariant SIFT descriptor and isomorphic graph representation. The performance of feature level fusion has verified by two distance metrics namely, K-NN and normalized correlation metrics. Normalized correlation metric is found to be superior to K-NN metric for all the proposed methods. The proposed feature fusion has evaluated with two different multibiometrics databases and a comparative study has been presented with another fusion approach of different paradigm.

\section{References}

1. Kisku, D.R., Gupta, P., Sing, J.K.: Feature Level Fusion of Biometric Cues: Human Identification with Doddington's Caricature. In: International Conference on Security Technology, CCIS, pp. 157-164. Springer, Heidelberg (2009)

2. Rattani, A., Kisku, D.R., Bicego, M., Tistarelli, M.: Feature Level Fusion of Face and Fingerprint Biometrics. In: 1st IEEE International Conference on Biometrics, Theory, Applications and Systems, pp. 1-6. IEEE Press, Los Alamitos (2007) 
3. Yao, Y.-F., Jing, X.-Y., Wong, H.-S.: Face and Palmprint Feature Level Fusion for Single Sample Biometrics Recognition. Neurocomputing 70(7), 1582-1586 (2007)

4. Ross, A., Govindarajan, R.: Feature Level Fusion using Hand and Face Biometrics. In: SPIE Conference on Biometric Technology for Human Identification II, pp. 196-204 (2005)

5. Fu, Y., Ma, Z., Qi, M., Li, J., Li, X., Lu, Y.: A Novel User-specific Face and Palmprint Feature Level Fusion. In: 2nd International Symposium on Intelligent Information Technology Application, pp. 296-300 (2008)

6. Yan, Y., Zhang, Y.-J.: Multimodal Biometrics Fusion using Correlation Filter Bank. In: International Conference on Pattern Recognition, pp. 1-4. IEEE Press, Los Alamitos (2008)

7. Kisku, D.R., Rattani, A., Grosso, E., Tistarelli, M.: Face Identification by SIFTbased Complete Graph Topology. In: 5th IEEE International Workshop on Automatic Identification Advanced Technologies, pp. 63-68. IEEE Press, Los Alamitos (2007)

8. Jain, A.K., Feng, J.: Latent Palmprint Matching. IEEE Transactions on Pattern Analysis and Machine Intelligence 31(6), 1032-1047 (2009)

9. Whitney, H.: Congruent Graphs and the Connectivity of Graphs. Am. J. Math. 54, 160-168 (1932)

10. Zhang, O., Couloigner, I.: A New and Efficient K-medoid Algorithm for Spatial Clustering. In: International Conference on Computational Science and Its Applications, pp. 181-189 (2005)

11. Lowe, D.G.: Object Recognition from Local Scale-invariant Features. In: International Conference on Computer Vision, pp. 1150-1157 (1999)

12. Theodoridis, S., Koutroumbas, K.: Pattern Recognition, p. 635

13. Horiuchi, T.: Colorization Algorithm using Probabilistic Relaxation. Image and Vision Computing 22(3), 197-202 (2004)

14. Dasarathy, B.V.: Nearest neighbor (NN) norms: NN Pattern Classification Techniques (1991)

15. Kumar, A., Wong, D.C.M., Shen, H.C., Jain, A.K.: Personal Verification using Palmprint and Hand Geometry Biometric. In: 4th International Conference on Audioand Video-Based Biometric Authentication, pp. 668-675 (2003)

16. Kisku, D.R., Tistarelli, M., Sing, J.K., Gupta, P.: Face Recognition by Fusion of Local and Global Matching Scores using DS Theory: An Evaluation with Uni-classifier and Multiclassifier Paradigm. In: IEEE Computer Vision and Pattern Recognition (CVPR) Workshop on Biometrics, pp. 60-65 (2009)

17. Ribarí, C.S., Fratríc, I.: A Biometric Identification System Based on Eigenpalm and Eigenfinger Features. IEEE Transactions on Pattern Analysis and Machine Intelligence 27(11), 1698-1709 (2005)

18. Dubes, R., Jain, A.K.: Clustering Techniques: The User's Dilemma. Pattern Recognition 8(4), 247-260 (1976)

19. Rokita, J., Krzyzak, A., Suen, C.Y.: Multimodal Biometrics by Face and Hand Images taken by a Cell Phone Camera. International Journal of Pattern Recognition and Artificial Intelligence 22(3), 411-429 (2008)

20. Rattani, A., Kisku, D.R., Logario, A., Tistarelli, M.: Facial Template Synthesis based on SIFT Features. In: 5th IEEE International Workshop on Automatic Identification Advanced Technologies, pp. 69-73 (2007)

21. Zhang, D., Kong, W.-K., You, J., Wong, M.: On-line Palmprint Identification. IEEE Transactions on Pattern Analysis and Machine Intelligence 25(9), 1041-1050 (2003) 